\title{
Internet of things based industrial environment monitoring and control: a design approach
}

\author{
Alaa Abdulhady Jaber ${ }^{1}$, Firas Khalil Ibrahim Al-Mousawi², Hayder Sabeeh Jasem ${ }^{3}$ \\ ${ }^{1}$ Mechanical Engineering Department, University of Technology, Iraq \\ ${ }^{2} \mathrm{Al}$-Mustansiriyah University, Iraq \\ ${ }^{3}$ University of Technology, Iraq
}

\begin{tabular}{l} 
Article Info \\
\hline Article history: \\
Received Jan 15, 2019 \\
Revised Jun 25, 2019 \\
Accepted Jul 5, 2019 \\
\hline
\end{tabular}

\section{Keywords:}

Blynk platform

Environment monitoring

Internet of things

NodeMCU

\begin{abstract}
In this research an internet of things (IoT) system is designed for the purpose of industrial environment monitoring and control. The system is mainly composed of control and sensor units. Control unit has the responsibility of managing the received data form the sensor unit and then executing the developed control algorithm based on the measured parameters. NodeMCU development kit is used as the core of the control unit. The senor unit contains gas and temperature sensors utilized for measuring the temperature and concentration of toxic gases in the monitored space. A buzzer has also been embedded in the sensor unit for alerting the occupants acoustically in the danger situations. If the monitored temperature gets higher or lower than the set levels, the air conditioning system will be automatically operated. Similarly, the fan (ventilation) system will be operated if the level of toxic gases becomes high. A mobile application, based on Blynk platform, is then developed to enable the wireless monitoring and control of the environment by the in charge people. In addition to the automatic and wireless control the manual control capability is considered in the developed IoT system.
\end{abstract}

Copyright $\odot 2019$ Institute of Advanced Engineering and Science. All rights reserved.

\section{Corresponding Author:}

Alaa Abdulhady Jaber,

Mechanical Engineering Department,

University of Technology,

Baghdad, Iraq.

Email: 20039@uotechnology.edu.iq

\section{INTRODUCTION}

Monitoring environmental parameters, such as temperature, humidity and the concentration of toxic gases, in residential or industrial medium is of great importance for keeping the occupants safe and comfortable. Many previously conducted researches have been focusing on developing different hardware and software configurations for environment monitoring and control. For instance, researchers have developed a wireless sensor network (WSN) system for environment monitoring in an instant coffee factory [1]. In this research a wireless sensor node composed of four layers and based on 8051microcontroller from Analog Devices and a Spartan 3 FPGA from Xilinx have been constructed. Of these four layers there are two sensor layers; the first one is adopted to manage the water quality measurements and the second is to measure the emitted gas level. The measured parameters are $\mathrm{pH}$, temperature of the waste water and the concentration level of number of emitted gases, such as carbon monoxide (CO), Sulphur dioxide $\left(\mathrm{SO}_{2}\right)$, and nitric oxide (NO). Another paper proposed an agricultural environment monitoring server system that integrates environmental and soil sensors, GPS module and CCTV into a device to collect information for monitoring the environment at crop plantations [2]. The system provides real-time environmental monitoring and various application services based on the collected information. In term of electrical power, the proposed server system was provided with a solar cell module as its power source, 
thus it can work without supplying any external power. However, the problem faced in the developed system was the server's consumed power is significantly larger than the power generated in the solar cell module, because of this the operating time was quite short. To solve this problem, the server was rebuilt to implement the system with low power embedded board modules, and this has increased the operating time of the proposed system. Similarly, greenhouse temperature monitoring and control system has been developed by Xiao, et al. [3]. Wireless data transmission capability has been added to the system using ZigBee wireless communication protocol. The temperature acquisition and then wirelessly transmitting it to a terminal node were achieved utilizing two CC2530 node, which is an integrated development environment module.

Jinsong, et al. [4] designed a small, low-cost, easy to layout urban environmental quality monitoring terminal, and multi-terminal constitutes a distributed network. The designed system has the capability of detection air pollution gas, inhalable particle and meteorological parameters. Based on the data received from the terminals, the data platform center can monitor and record the real-time dynamic data. Then, it establishes monitoring system database, analysis the real-time dynamic data and predict the regional environmental pollution situation and development trends, which provide a scientific basis to effectively control the environmental conditions. Another interesting research is about developing an automatic air temperature and humidity monitoring system in the cultivation of oyster mushroom based on Arduino Uno microcontroller [5]. The developed system was integrated with wireless capability (Bluetooth and Wi-Fi) and an Android application for remote control and data displaying. Depending on the measured temperature and humidity values the microcontroller turn on the pump automatically to pump water in order to lower the room temperature if it is higher than normal, or the light will be turned on to heat the room if gets cold. Also, other researchers have been focusing on developing smart environment monitoring and control appliance for homes [6]. In this research a smart fan that is able to monitor the room temperature and based on it its speed is either increased or decreased automatically has been built. The developed fan is based on ESP8266 microcontroller, DHT22 temperature sensor and HC-SR04 ultrasonic sensor. The ultrasonic sensor is utilized for detecting people availability in the room. However, the researchers have carried out interviews with number of people that have been asked to test the developed fan and they have shown high satisfaction, due to its advantages in energy reduction and comfortability. Generally, a study has been conducted by the Health Effect Institute in 2016 concluded that exposing people to pollutant air for a long-term was the reason for death 6.1 million people due to casing different diseases, such as, heart attacks, strokes and lung cancer [7].

In the present research, however, an industrial environment monitoring and then controlling it via powering on or off the air conditioning and ventilation systems by using Internet of things (IoT) concept is going to be thoroughly investigated, as discussed in the following section. The design approach and the used electronic components are thoroughly discussed.

\section{GENERAL DESCRIPTION OF THE DEVELOPED SYSTEM}

The proposed system is mainly composed of four parts; these are control unit, sensing unit, the smartphone application for remote monitoring and control, and the embedded code that is used for system management. The main purpose of these four parts together is to automate the operation of the ventilation, heating and cooling systems inside the industrial facilities and labs. This, in addition to innocent any environmental danger, will help in reducing the consumed energy and avoiding the forgetfulness errors, such as forgetting turning on the above mentioned systems when they are needed. Also, the capability of alerting the people who are in charge to take an action or to evacuate the building when there is a high level of risk is considered to be integrated in the proposed system. The stated four parts of the designed system are deliberated in details in the subsequent sections.

\section{CONTROL UNIT DESIGN}

Any control unit is mainly built based on a microcontroller module and different sensors, actuators and displaying device are interfaced to it. Thus, the main components that have been used in the control unit development and how they are connected together are discussed as follows.

\subsection{NodeMCU development kit}

The core of the developed electronic system in this research is NodeMCU version 2, it serves as its main controller unit. It handles the wireless connectivity, connection to load, communication with peripherals, control and display processes. However, there are many microcontrollers are available in the market, but this one was chosen due to its small size $(49 \times 24.5 \times 13 \mathrm{~mm})$, affordable price, low power consumption and extent of community support [8]. The NodeMCU module uses ESP8266 Wi-Fi module, 
integrated with 32-bit RISC TenSilica L106 microcontroller running at $80 \mathrm{MHz}$; this creates a low-cost and low-power consumption development platform for embedded and IoT applications [9, 10]. NodeMCU is an open source platform and can be programmed using Arduino IDE (using micro USB) so the different libraries used by Arduino can be run on it. Figure 1 shows the pins diagram of the NodeMCU along with its various inputs/outputs capabilities. It has 16 general purpose input-output (GPIO) ports, 13 of them can be configured to be pulse-width modulation (PWM) sources. Also, it contains Inter-Integrated Circuit (I2C) protocol, 10-bit analog-to-digital converter (ADC), Serial Peripheral Interface (SPI) protocol, UART on dedicated pins, in addition to a transmit-only UART can be enabled on GPIO2.

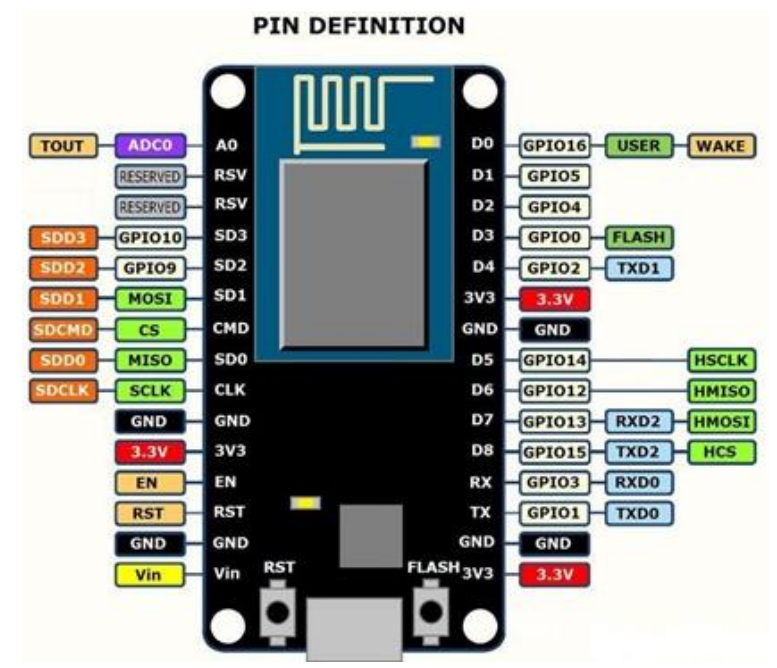

Figure 1. NodeMCU development kit

\subsection{Display screen}

In order to show the status of ventilation and air conditioning equipment and displaying the acquired sensor information, such as gas temperature, ambient temperature and humidity, the OLED screen has been utilized. It is an organic light emitting diode (OLED), has a flat light emitting technology, made of a series of organic thin films placed between two conductors and having dimensions of 128 x 64 [10, 11]. It consumes low power, has higher image quality and can be driven by SSD1306 single chip driver. The OLED display is interfaced with the NodeMCU board with the application of I2C peripheral interface protocol. The I2C protocol is a 2-wire serial communication standard comes with serial data line (SDA) and serial clock line (SCL), which allows a serial transmission of data/commands between the OLED display and the NodeMCU board so a bit is sent on each clock pulse. As shown in Figure 2, connecting the screen with the microcontroller is simple and can be achieved using only 4 pins, by which the GPIO5 (D1) and GPIO4 (D2) on the NodeMCU board are configured with the SCL and SDA line of OLED display, respectively. After setting up the hardware connections the library for the SSD1306 driver needs to be downloaded from (https://github.com/acrobotic/Ai_Ardulib_SSD1306) and added to the Arduino IDE.

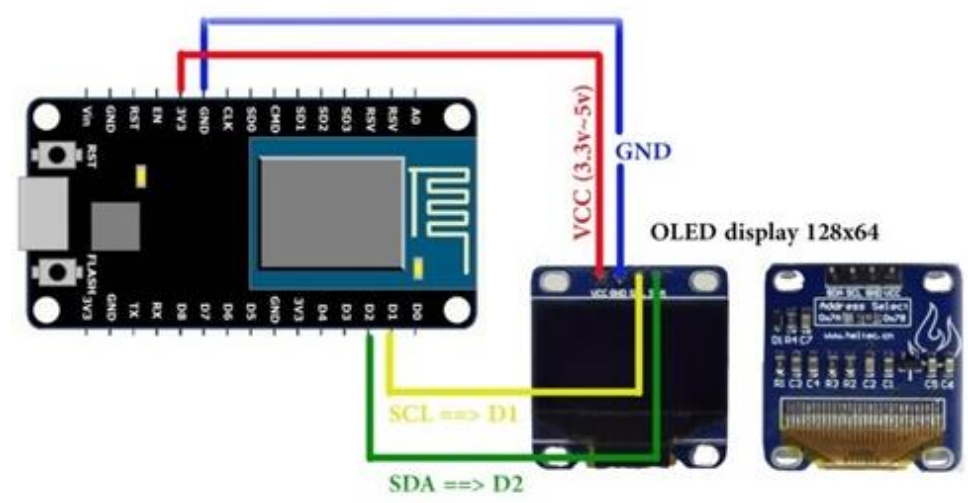

Figure 2. Interfacing the OLED screen to NodeMCU 


\subsection{Temperature and humidity sensor}

To measure the temperature and humidity of the ambient environment DHT22 digital sensor is used. This sensor was chosen among others, such as DHT11, SHT71, since it is cost effective, consumes less power, comes with long term stability, gives calibrated digital signal and, importantly, more accurate [10]. Its temperature measurement range is $0-50{ }^{\circ} \mathrm{C}$ with an accuracy of $\pm 2 \mathrm{C}^{\circ}$ and relative humidity range is $20-95 \%$ with an accuracy of $\pm 5 \%$. The industry of DHT22 is based on the piezo resistive technology and it also has 4 pins of which 2 pins uses I2C protocol (SCL and SDA). The sensor's VCC and GND pins are connected to $3.3 \mathrm{~V}$ and GND of NodeMCU while its data pin is connected to GPIO0 (D3) pin on the board, respectively, as shown in Figure 3. As previous, the DHT22 library is required to be add to the Arduino IDE after it gets downloaded from (https://github.com/adafruit/DHT-sensor-library).

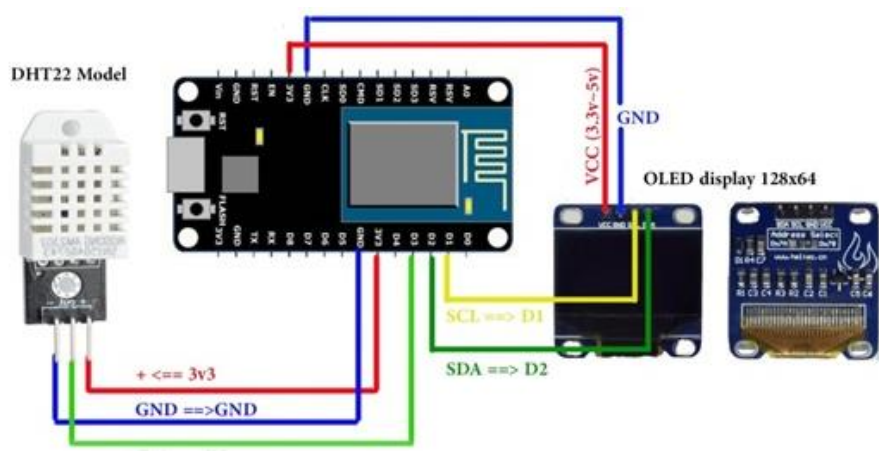

Figure 3. Interfacing the DHT22 temperature sensor to NodeMCU

\subsection{Appliances control}

A relay is an automatic and electrically operated switch that uses a small electric current to control larger current. It has control and controlled systems, which are also known as the input and output circuits respectively, however, the two circuits are electrically separated and magnetically attached. Thus, it is a very useful device for permitting one circuit to control a totally isolated one [12,13]. Mainly, there are two types of relays: electromechanical relay, which has an electromagnet containing a wire coil that becomes a temporal magnet when electricity flows through it, leading to actuation a current passing switch, and solid state relay (SSR), which is based on active semiconductor devices that uses light instead of magnetism to actuate the switch. The latter has no moving parts, no contact bounce, and fast switching speed compared with the electromechanical relays. Here, two channels SSR have been utilized to control the $220 \mathrm{~V}$ supplied power, one is for the air conditioning appliance and the other is for ventilation appliance, to switch them on and off based on the monitored environmental parameters. The channels are connected to GPIO12 (D6) and DPIO13 (D7), respectively as shown in Figure 4.

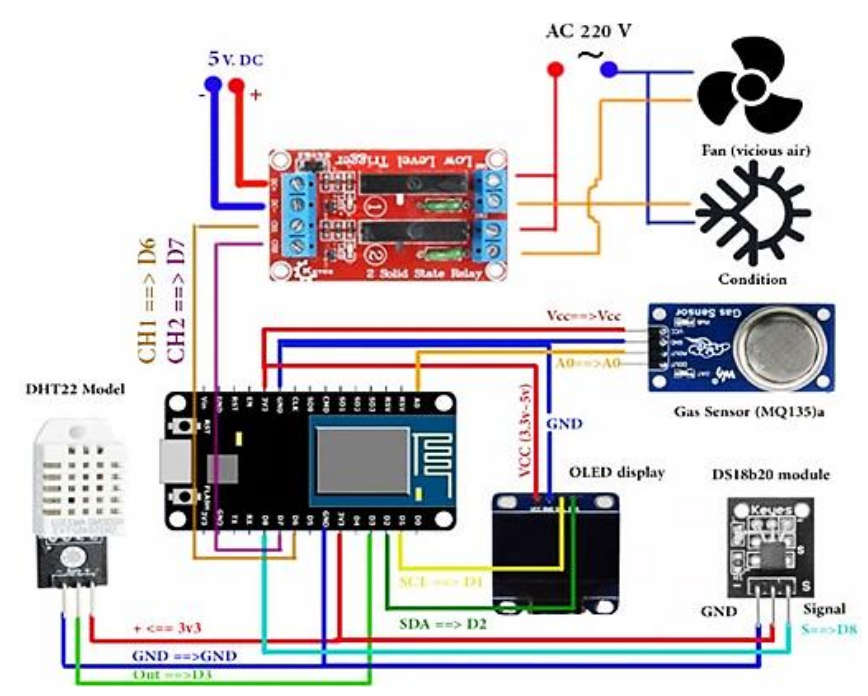

Figure 4. Interfacing a solid state relay to NodeMCU 


\subsection{Manual control capabilities}

In addition to the appliance automatic control, user manual control capability has been added to the developed system. This allows manual control of the ventilation and air conditioning systems based on sensors readings and the vision of the people who are responsible for applying the occupational safety systems. Thus, three push buttons have been connected to NodeMCU. Two buttons are configured to power on and off the air conditioning and ventilation systems respectively, while the third is configured to update the sensors data. However, continuous pressing state of the buttons has been considered to execute their related codes, this is to avoid mistaken presses case. The buttons are connected to NodeMCU via GPIO1 (Tx), GPIO3 (Rx) and GPIO2 (D4), respectively as shown in Figure 5.

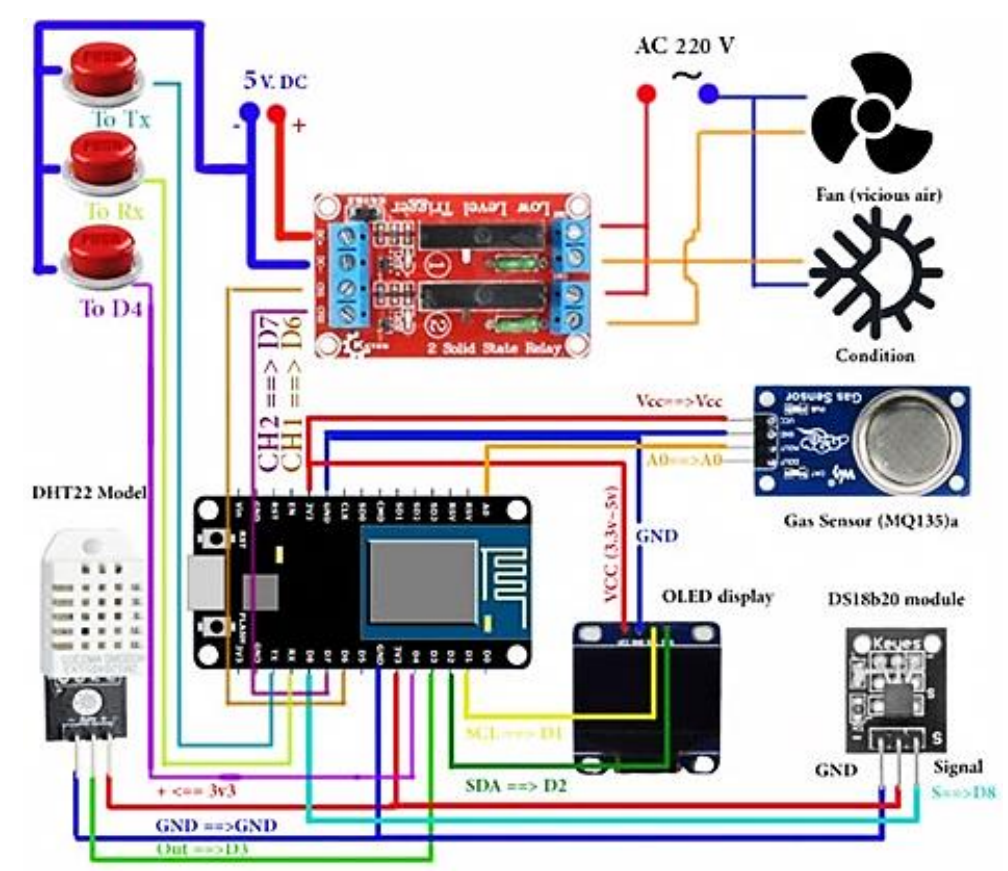

Figure 5. Interfacing three push buttons to NodeMCU

\section{SENSING UNIT DESIGN}

As the name refers, the sensor node includes number of sensors that are employed for measuring number of vital environmental variables. The acquired information by the sensor node are then communicated back to the control unit and based on the set conditions the designed control algorithm is executed. The components of the sensor node are deliberated in the following.

\subsection{Gas concertation level sensor}

To monitor the percentage of harmful gases in buildings and offices the MQ-135 sensor is widely used. MQ-135 is small, analog sensor, has high sensitivity to Ammonia $\left(\mathrm{NH}_{3}\right)$, Carbon dioxide $\left(\mathrm{CO}_{2}\right)$, Sulfide, Alcohol, Benzene, and also sensitive to smoke. Its working principle is based on employing Tin dioxide $\left(\mathrm{SnO}_{2}\right)$, which has a lower conductivity in clear air and as the concentration of the polluting gases increases the conductivity of the sensor increases too. The sensor detection range is 10-1000 ppm (particles per million) and the standard detecting conditions is when the supply voltage is $5 \mathrm{~V} \pm 0.1$, the ambient temperature is $20 \mathrm{C}^{\circ} \pm 2$ and the humidity is $65 \% \pm 5 \%$. The gas concentration value is found by converting the corresponding analog voltage output of the MQ-135 sensor using the following formula [14]. Where $V_{c c}$ is the sensor supply voltage, $V_{O}$ is the sensor output voltage, $R_{L}$ is the load resistance, $R_{O}$ is the sensor resistance in the standard detecting conditions, and $c$ and $m$ are the slope constants calculated from the sensitivity chart of MQ-135 Datasheet [15].

$$
p p m=\left(\left(\frac{V_{c c}}{V_{o}}-1\right) *\left(\frac{R_{L}}{R_{O}} * 10^{-C}\right)\right)^{\frac{1}{m}}
$$


The MQ-135 sensor has the capability to output the measurement results in analog or digital representation. If digital output is used, the library of MQ-135 sensor has to be downloaded and add to Arduino IDE, whereas it is not needed if analog output is considered, which is the case in this research. Figure 6 illustrates how this sensor is interfaced to the NodeMCU; three wires have been utilized, two of them are for supplying the power to the sensor and the third is for transferring the sensor output to NodeMCU, which is connected to analog input A0.

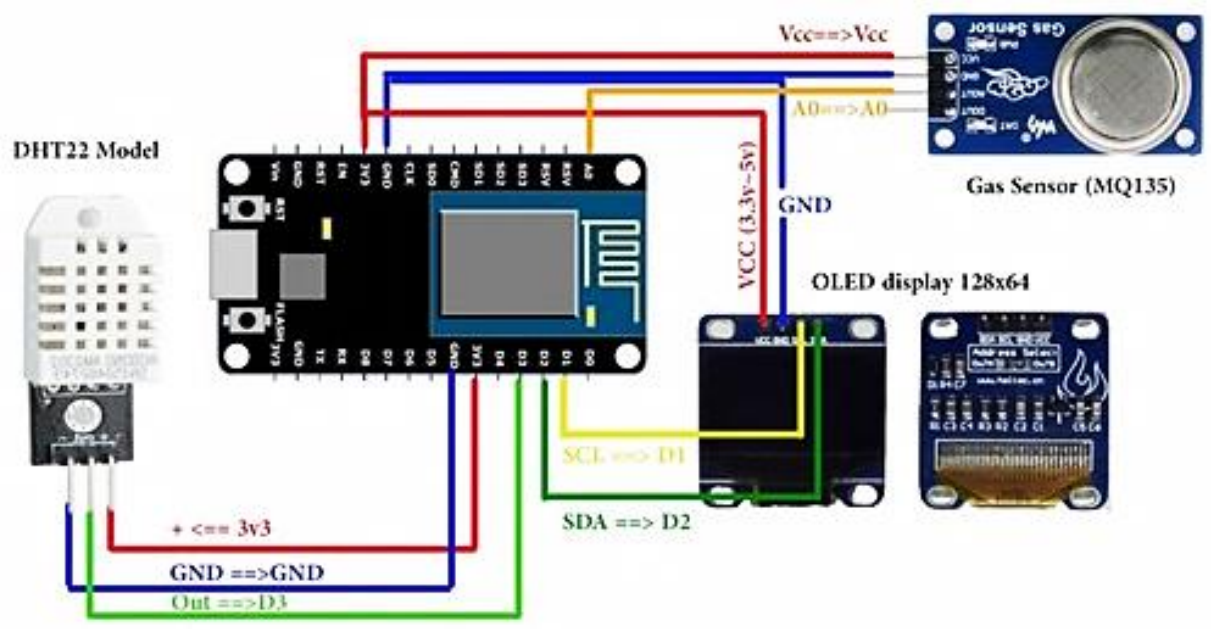

Figure 6. Interfacing the MQ-135 gas sensor to NodeMCU

\subsection{Gas temperature sensor}

The utilized sensor for gathering the gas temperature is DS18B20, which is a precise, digital sensor, produced by Dallas Semiconductor Company and it uses a 1-wire protocol for communication with the microcontrollers. The sensor has the capability of temperature sensing and the analogue to digital (ADC) data conversion $[16,17]$. Its measurement range is from $-55 \mathrm{C}^{\circ}$ to $+125 \mathrm{C}^{\circ}$ with high precision reaches to $0.0625 \mathrm{C}^{\circ}$ and expanded measurement uncertainty of $\pm 0.5 \mathrm{C}^{\circ}$, however, the measured temperature value can be shown in 9 to 12 bits $[18,19]$. In order to interface this sensor with NodeMCU two libraries, which are OneWire and DallasTemperature, have to be downloaded from (https://github.com) and add to Arduino IDE. The data pin of the sensor is connected to GPIO15 (D8) as shown in Figure 7. However, for accurate measurement result this sensor along with the gas sensor have to be installed either on the celling or on one of the corners of the monitored space. For acoustically alerting the occupants in the dangerous situations a small buzzer has also been installed with these two sensors.

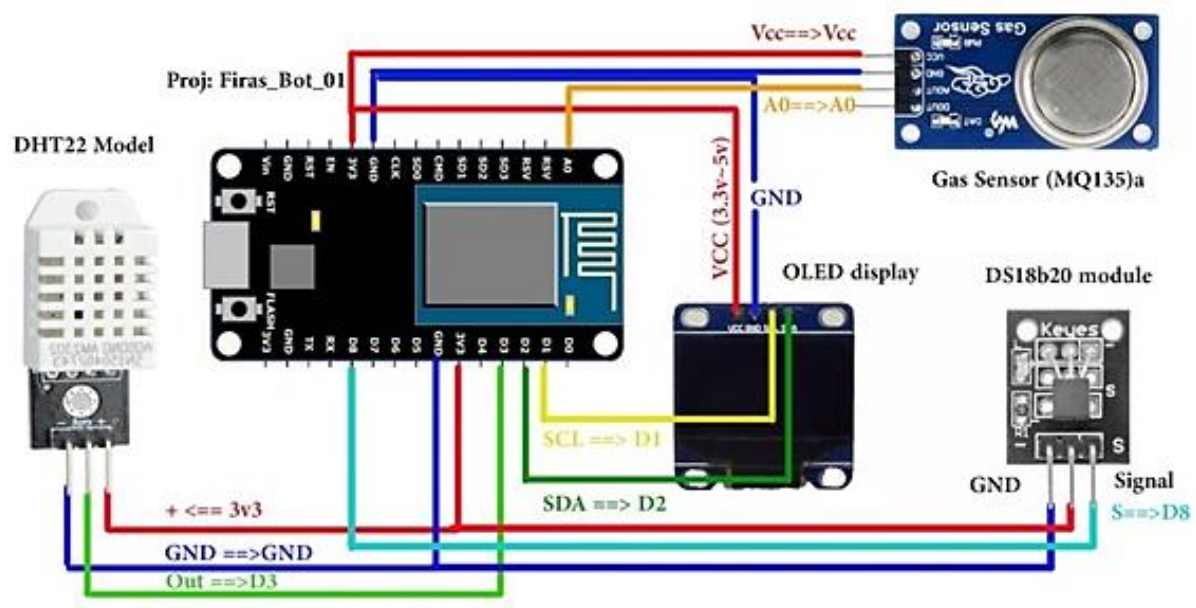

Figure 7. Interfacing the DS18b20 temperature sensor to NodeMCU 


\section{SMARTPHONE APPLICATION}

Blynk is an android and ISO platform, commonly used to easily control Arduino, Raspberry Pi and many others microcontrollers, such as NodeMCU in this research, over the Internet with minimal coding requirements. It is a digital dashboard where a graphical user interface for a project can be created by just dragging and dropping the needed icons. Blynk has its own server, which is an open-source one based on Java server. It is responsible for forwarding messages between Blynk mobile application and the utilized microcontroller boards. Also, Blynk has many libraries for the mentioned hardware, these libraries enable the communication with the dedicated sever and are responsible for processing the in- and out-coming commends as shown in Figure 8.

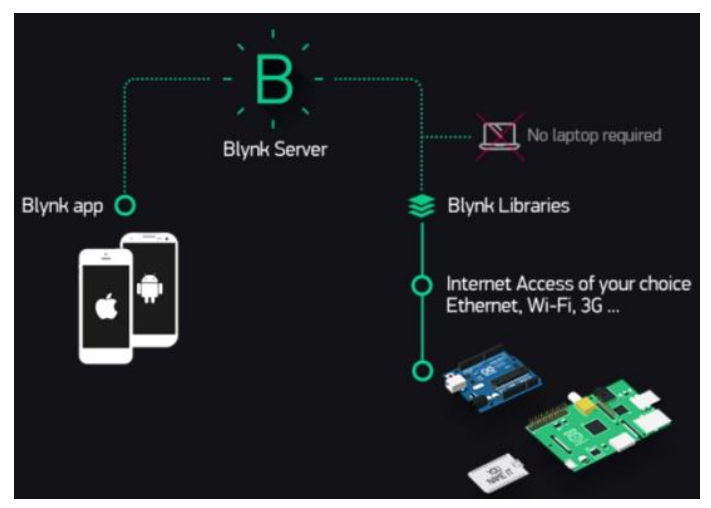

Figure 8. Blynk platform working principle [20]

However, to develop a graphical user interface (GUI) on a smartphone the Blynk application has to be downloaded and installed first. After installing it a new project has to be created and the microcontroller type that it requires to communicate with (here NodeMCU) has to be selected. Then, Blynk will issue an authorization number, which is then included in the developed Arduino sketch in order to ensure the wireless connection between the smartphone and NodeMCU. On the NodeMCU side the library "BlynkSimpleEsp8266" has to be downloaded from (https://github.com/blynkkk/blynk-library) and included in the sketch. This library will be in charge of setting up and configuring the wireless communication. Then, the Wi-Fi name and password, in addition to the authorisation number, are added to the sketch as below.

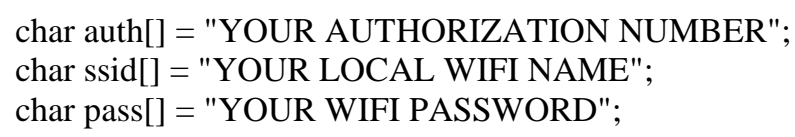

In this research the developed Blynk application has four capabilities which are (1) receiving data from the control unit in order to be viewed on the smartphone (2) displaying warning messages to indicate the abnormalities in the monitored parameters (3) lighting up number of LEDs if substantial changes in the monitored parameters are detected and (4) has control buttons that can be used to turn on and off the ventilation and air conditioning systems, as illustrated in Figure 9.

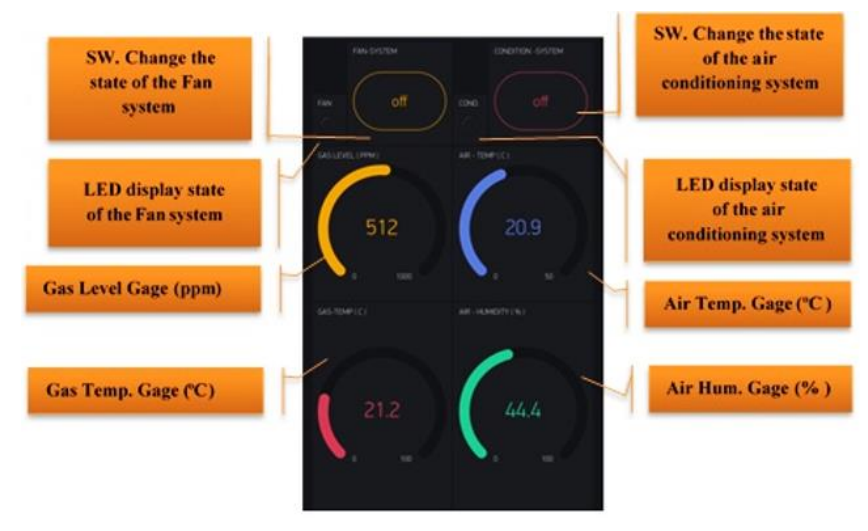

Figure 9. The developed smartphone application based Blynk platform 


\section{THE DEVELOPED EMBEDDED CODE}

Figure 10 (refer appendix) illustrates the block diagram of the developed code for the proposed environment monitoring and control system. As it is obvious, the program is downloaded on the NodeMCU after it is being synthesized using Arduino IDE. When the control unit is powered on, the code first starts initialization and setting up the required input and output pins and then displaying the saved standard parameters onthe OLED screen. Establishing the wireless connectivity between the ESP8266 module and the available Wi-Fi network represents the second stage in the code. Acquiring sensors readings and presenting them on the OLED screen and at the same time sending them wirelessly to the developed mobile application considers the following step. However, the code is continuously monitors the updating button in case it gets constant press for one second in order to update the displayed readings on the screen. If the button has not been pressed, the code will update the screen every 30 seconds. The code sends an order to power on the air conditioning and/or fun systems if their related buttons (number 7 and 8 in Figure 11 in the following section) are pressed for one second too. This step is known as the manual control mode. Hence, the code follows the automatic control mode if button press is not detected. The automatic mode mainly depends on the measured temperature and toxic gas level. If the measured temperature is less than $18 \mathrm{C}^{\circ}$ the air conditioning system is operated in the heating mode whereas it is operated in the cooling mode if the measured temperature is higher than $29 \mathrm{C}^{\circ}$. Otherwise, the conditioning system is powered off if the temperature is higher than $18 \mathrm{C}^{\circ}$ and less than $29 \mathrm{C}^{\circ}$.

If the detected toxic gases level is less than $400 \mathrm{ppm}$, the ventilation (funs) system is powered off and a message "gas normal" is displayed on the screen and simultaneously sent to the mobile application. A warring message stating "gas abnormal" is presented on the screen and concurrently forwarded to the mobile if the toxic gases level is higher than $400 \mathrm{ppm}$ and less than $850 \mathrm{ppm}$. However, when the toxic gases level is higher than $850 \mathrm{ppm}$, the environment is considered very dangerous for the occupants and thus a very cautionary message declaring "gas danger" is presented on the screen and transmitted to the mobile, respectively.

\section{SYSTEM PACKAGING}

Packaging the developed hardware system represents the final stage in the hardware system design. Thus, in order to keep the components of the designed system safe, connected together and stable and also for future distribution and usage a customized cover made of plastic has been configured for this purpose. Figure 11 shows the final product of this research, by which the main components of the developed system are numbered. These components are listed and discussed as follows.

1. DHT22 digital temperature and humidity sensor: it is previously discussed.

2. Antenna socket of the ESP8266 Wi-Fi module: it is used to exchange data between the control unit and the mobile device.

3. AC Power plug: powering the control unit is done by plugging it to the main AC power supply in the monitored facility using three pins, type G plug. The supplied current and voltage by this type of plugs are $13 \mathrm{~A}$ and $220-240 \mathrm{~V}$, respectively. So, an internal power convertor, to transform the supplied voltage down to the needed level of power, along with LM7905 voltage regulator, to maintain the voltage in a circuit comparatively close to the desired value, have been utilized to provide the NodeMCU with $5 \mathrm{~V}$, DC current. Accordingly, NodeMCU provides the relays with the control current and the sensors with the required voltage for operation. Moreover, the AC power plug is also used for powering the air conditioning and fan systems.

4. Upper socket: this socket outputs $2 \mathrm{~A}$ and $220-240 \mathrm{~V}$ and used for powering the air conditioning system.

5. Front socket: it also outputs $2 \mathrm{~A}$ and $220-240 \mathrm{~V}$, but used for powering the fan system.

6. Updating button (red): it is used to update the sensor readings on the display screen.

7. A green button: it is utilized for manually operating the air conditioning system.

8. A blue button: it is utilized for manually operating the fan system.

9. USB type C: to be used for uploading the developed sketch (software), based on Arduino IDE, on the NodeMCU.

10. OLED 64 x 128 screen: it is utilized for showing the sensors reading, the situation of the monitored space and the status (on or off) of the air conditioning and fan systems, as previously illustrated.

11. Power LED: it is exploited to indicate the operation state of the control unit.

12. Extra output socket: this socket outputs $1 \mathrm{~A}$ and $12 \mathrm{~V}$ and it can be employed for the purpose of future development or for operating required additional device. 
13. RJ45 socket: it used to power the sensing unit via RJ45 cable. Hence, RJ45 socket is a connector commonly utilized for Ethernet networking. It has eight pins and, accordingly, meaning that the RJ45 cable containing eight separated wires, which correspondingly forming four pairs for powering the gas and temperature sensors and the buzzer in the sensing unit and one pair is left free.

14. The sensor unit: the main components of this unit and how they are connected to the NodeMCU have been previously discussed in Section 4.

15. The MQ-135 gas sensor: it is powered from control unit and it communicates the acquired data to back to NodeMCU.

16. Alerting buzzer: it is utilized to produce sound when the system is detecting an environmental abnormality in the monitored space.

17. DS18B20 temperature sensor: it has also been previously discussed.

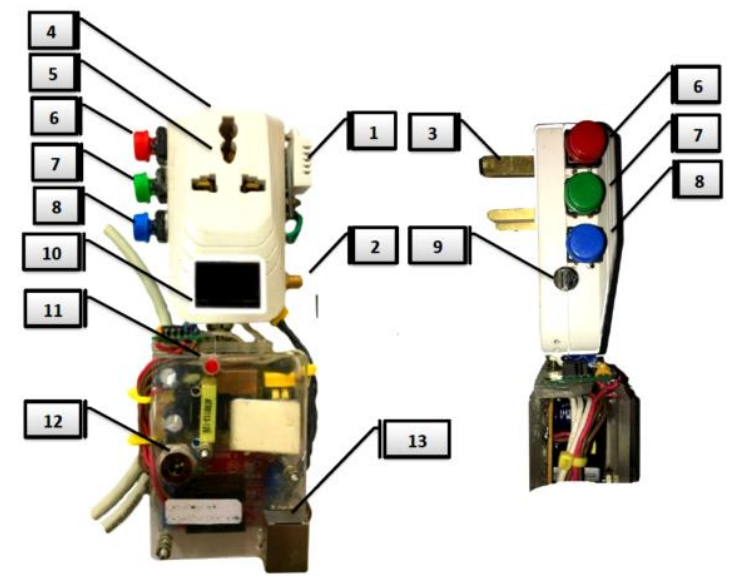

(a)

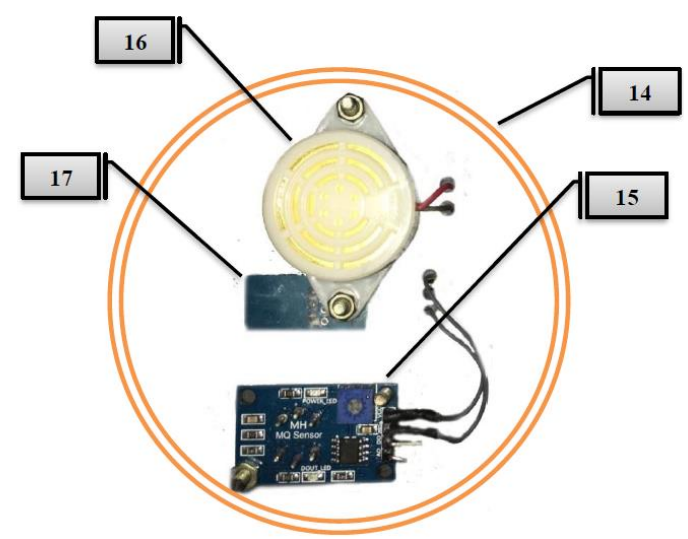

(b)

Figure 11. The developed electronic system, a) The control unit, b) Sensor unit

\section{CONCLUSION}

This paper has addressed the design and construction of an electronic system based on Internet of things (IoT) concept. The main aim of the designed IoT system, although it is not limited to this, is to monitor and then control the industrial environments, such as factories, or even labs. Detailed description of the electronic evolution has been provided in this research. Control and sensor units represent the main parts of the designed system. Low cost microcontroller, sensors and other electronic components have been utilized in this research. These include NodeMCU microcontroller, which uses ESP8266 Wi-Fi module, gas sensor for air quality evaluation, temperature sensors for measuring the ambient and gas temperatures and small display screen. The sensor unit is designed to be installed on the celling or wall of the monitored space and it gets electrically powered from the control unit via wire connection. To overcome the power matter of the control unit it has been designed to take its power directly from a power plug in the monitored space. Monitoring and control algorithm has been developed and then being coded and uploaded on the NodeMCU, 
which considers the core element of the system, using Arduino IDE. To allow the remote and wireless monitoring and control option to the responsible people a mobile application has been developed. The proposed algorithm has also deliberated alerting the in charge people in the abnormal situations using mobile messaging. In addition to automatic and wireless control capabilities manual control function has been enabled on the system. The final product has then been enclosed using custom built plastic box in order to be ready for use and work and to keep it safe. However, the utilized electronic components have individually been tested, thus, the next step is to conduct a real test by using the developed IoT system in an industrial environment and then analyse the captured data.

\section{REFERENCES}

[1] J. Valverde, V. Rosello, G. Mujica, J. Portilla, A. Uriarte, and T. Riesgo, "Wireless Sensor Network for Environmental Monitoring: Application in a Coffee Factory," International Journal of Distributed Sensor Networks, vol. 8, pp. 638067, 2011.

[2] J. Hwang, C. Shin, and H. Yoe, "Study on an Agricultural Environment Monitoring Server System using Wireless Sensor Networks," Sensors (Basel, Switzerland), vol. 10, pp. 11189-11211, 2010.

[3] M. Xiao, J. Liang, and Y. Shi, "ZigBee-based Temperature Controlling System for Agricultural Greenhouses," in 2018 5th International Conference on Systems and Informatics (ICSAI), 2018, pp. 526-531.

[4] D. Jinsong, W. Wei, G. Jie, and C. Rigang, "Distributed intelligent urban environment monitoring system," IOP Conference Series: Earth and Environmental Science, vol. 113, pp. 012222, 2018.

[5] P. Sihombing, T. P. Astuti, Herriyance, and D. Sitompul, "Microcontroller based automatic temperature control for oyster mushroom plants," Journal of Physics: Conference Series, vol. 978, pp. 012031, 2018.

[6] K. Keeratiburt, "Automatic Speed Control and Turning ON/OFF for Smart Fan by Temperature and Ultrasonic Sensor," IOP Conference Series: Materials Science and Engineering, vol. 325, pp. 012022, 2018.

[7] P. Arroyo, J. Lozano, and J. I. Suárez, "Evolution of Wireless Sensor Network for Air Quality Measurements," Electronics, vol. 7, pp. 342, 2018.

[8] X. Bajrami and I. Murturi, "An efficient approach to monitoring environmental conditions using a wireless sensor network and NodeMCU," $e$ \& i Elektrotechnik und Informationstechnik, vol. 135, pp. 294-301, Jun 2018.

[9] A. Yasirli and S. Mukhammad Andri, "Improving Smart Home Concept with the Internet of Things Concept Using RaspberryPi and NodeMCU," IOP Conference Series: Materials Science and Engineering, vol. 325, pp. 012021, 2018

[10] R. K. Kodali and K. S. Mahesh, "Low cost ambient monitoring using ESP8266," in 2016 2nd International Conference on Contemporary Computing and Informatics (IC3I), 2016, pp. 779-782.

[11] N. T. Surajudeen-Bakinde, S. O. Ayodele, T. D. Oloruntoba, A. O. Otuoze, and N. Faruk, "Development of an Internet based prepaid energy meter," in 2017 IEEE AFRICON, 2017, pp. 1370-1373.

[12] N. Abraham, R. Ramar, and J. Ramachandran, "Self Regulating Power Saving System for Home Automation," in Artificial Intelligence and Evolutionary Computations in Engineering Systems, Singapore, pp. 121-132, 2018.

[13] T. Pan and Y. Zhu, "Intelligent Lock System Using Arduino," in Designing Embedded Systems with Arduino: A Fundamental Technology for Makers, ed Singapore: Springer Singapore, 2018, pp. 205-220.

[14] A. M, "Development of Gas Leakage Monitoring and Localization System in Pipelines using LabVIEW," International Journal of Applied Engineering Research, vol. 13, pp. 7868-7873, 2018.

[15] H. E. C. Ltd, "Technical Data for MQ-135 gas sensor," ed.

[16] M. Kovačić, M. Vražić, and I. Gašparac, "Bluetooth wireless communication and 1-wire digital temperature sensors in synchronous machine rotor temperature measurement," in Proceedings of 14th International Power Electronics and Motion Control Conference EPE-PEMC 2010, 2010, pp. T7-25-T7-28.

[17] J. Doolan Fernandes, F. Carvalho Souza, G. Cipriano Maniçoba, A. Salazar, and J. de Paiva, "Wireless Monitoring of Induction Machine Rotor Physical Variables," Sensors, vol. 17, pp. 2660, 2017.

[18] M. Jankovec and M. Topič, "Intercomparison of Temperature Sensors for Outdoor Monitoring of Photovoltaic Modules," Journal of Solar Energy Engineering, vol. 135, pp. 031012-031012-7, 2013.

[19] P. Li, J. Li, and J. Jing, "Wireless temperature monitoring system based on the ZigBee technology," in 2010 2nd International Conference on Computer Engineering and Technology, 2010, pp. V1-160-V1-163.

[20] https://www.blynk.cc/. 


\section{APPENDIX}

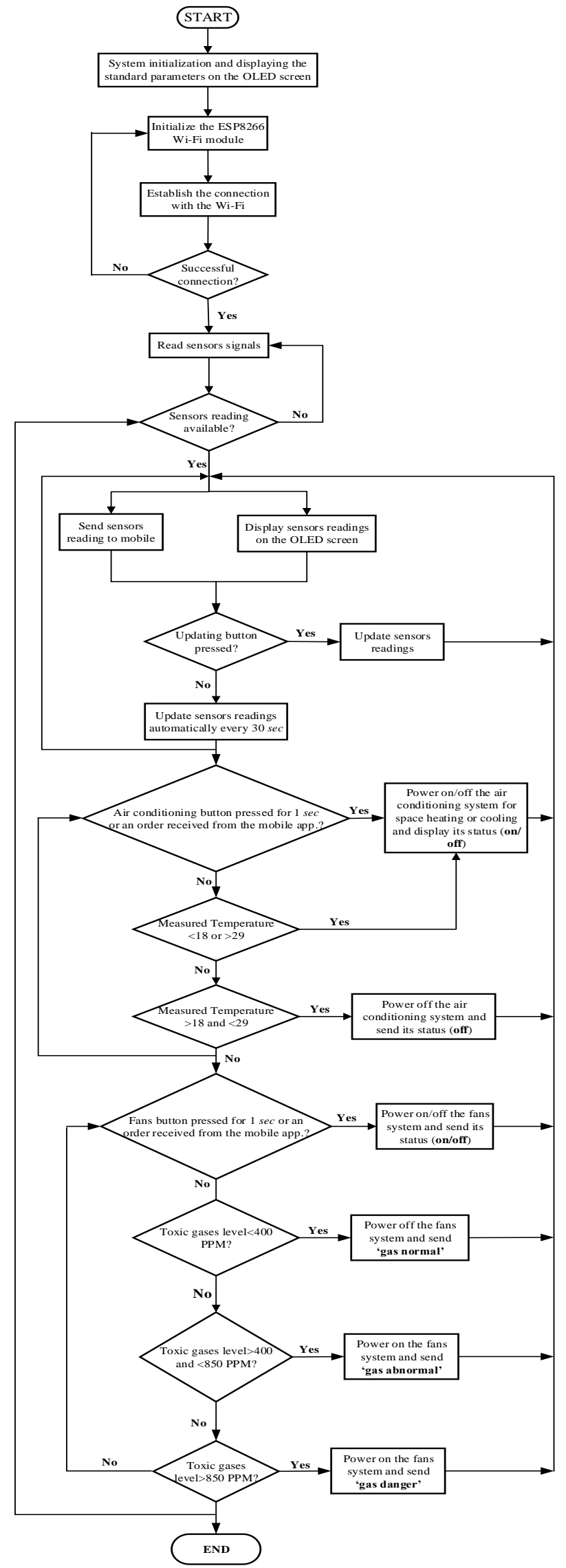

Figure 10. The developed embedded code 